\title{
Prevalence of Depression Symptoms in Outpatients with a Complaint of Headache
}

\author{
Robert A. Marlow, MD, MA, Cynthia L. Kegowicz, MD, and Kimberly N. Starkey, BA
}

Purpose: This case-control study was designed to determine whether adults who present to a primary care office with a chief complaint of headache have more reported symptoms of depression than adults presenting with other problems.

Metbods: Adult, English-speaking patients who presented to a primary care office with a chief complaint of headache were matched to adult patients of the same age and sex who presented with problems other than headache. All participants completed the PRIME-MD 9-item Patient Health Questionnaire as a screen for depression.

Results: A total of 200 participants entered the study. The mean age of the participants was 43.8 years (range, 18-87 years). Women constituted $84 \%$ of the participants. Of those patients who presented with headache, $32 \%$ had a likelihood of possible major depressive disorder compared with $12 \%$ in the patients presenting without headache.

Conclusions: Almost one-third of adult patients who present to a primary care office with a complaint of headache report moderate symptoms of depression when screened compared with approximately $10 \%$ of patients presenting with a complaint other than headache. Given such a high prevalence of these symptoms, primary care physicians should screen all adult patients who present with headache for depression. (J Am Board Fam Med 2009;22:633-637.)

It is estimated that more than $90 \%$ of adults experience at least one headache each year. Although the majority of those adults do not seek medical attention for this problem, headache is one of the top reasons for people to seek outpatient medical care. Depression is also a common disorder seen in the outpatient setting. In primary care, anywhere from $5 \%$ to $10 \%$ of outpatients have major depression. ${ }^{1,2}$ Patients who suffer from pain syndromes, especially chronic ones, have an even higher prevalence of depression. Therefore, outpatients who present to primary care physicians with a complaint

This article was externally peer reviewed.

Submitted 13 April 2007; revised 29 April 2009; accepted 1 May 2009.

From the Scottsdale Healthcare Family Medicine Residency Program, Scottsdale, Arizona.

Funding: none.

Prior presentation: Poster was presented at the 34th North American Primary Care Research Group Annual Meeting, Tucson, Arizona (October 16, 2006).

Conflict of interest: none declared.

Corresponding author: Robert A. Marlow, MD, MA, Scottsdale Healthcare Family Medicine, 7301 E. 2nd Street, Suite 210, Scottsdale, AZ 85251 (E-mail: rmarlow@shc.org).

\footnotetext{
See Related Commentary on Page 602.
}

of headache would probably have a significant prevalence of depression.

Chung and Kraybill ${ }^{3}$ reported in 1990 that $63 \%$ of patients who presented to their outpatient family practice with a chief complaint of headache had "depression" based on a score of $>50$ on the Zung Self-Rating Depression Scale. They concluded that headache was an important marker for depression. However, they did not measure the prevalence of depression in their outpatients who presented with complaints other than headache. In 1999 Mitsikostas and Thomas ${ }^{4}$ observed in a Veterans' hospital in Greece that patients seen for headache had a higher prevalence of depression (3.4\%), which they defined as "major depression" or "dysthymia," compared with healthy patients $(0.6 \%)$, which was a much lower prevalence than found in the previously cited study. Mitsikostas and Thomas ${ }^{4}$ used the Hamilton Rating Scale for Depression, and they did not assess depression in "nonhealthy" outpatients who presented with some complaint other than headache.

In 2002, when using a clinical interview to screen, investigators at a specialized headache center noted a significant association between migraine headaches and major depression and dysthymia in their outpatients. ${ }^{5}$ Lipton et $\mathrm{al}^{6}$ reported in 2000 
from a population-based sample of patients in the United States and the United Kingdom that 47\% of migraine patients had "depression" compared with $17 \%$ of control patients; these results were based on the use of the Primary Care Evaluation of Mental Disorders (PRIME-MD). These authors did not further define "depression." Neither of these 2 studies included patients with headaches other than migraine, nor were they located in a primary care setting. Because the symptoms of depression are often subtle and hard to verbalize, these symptoms may be overlooked in patients who present with headache as their chief complaint. ${ }^{7,8}$

Several instruments have been used in the primary care setting to screen for depression among adults. The most commonly used measures in outpatient settings are the Beck Depression Inventory; the Center for Epidemiologic Studies Depression Scale, Revised; the PRIME-MD Patient Health Questionnaire (PHQ-9); and the Zung Self-Rating Depression Scale. According to a recent systematic review of depression screening tools by Nease and Malouin ${ }^{9}$ the PHQ-9 is the best depression screening tool available for primary care. The purpose of our study was to screen, using the PHQ-9, for the prevalence of reported symptoms of depression in primary care outpatients who presented with a chief complaint of headache compared with outpatients who presented with chief complaints of problems other than headache.

\section{Methods}

\section{Instrument}

The PRIME-MD PHQ-9 is a self-administered instrument that scores each of 9 Diagnostic and Statistical Manual of Mental Disorders, Fourth Edition, criteria for major depression. The scores range from " 0 " (not at all) to " 3 " (nearly every day). The measure takes an average of 2 to 5 minutes to complete, has good reliability and validity, and has a relatively high positive predictive value for major depression. ${ }^{9-11}$ Kroenke et $\mathrm{al}^{10}$ assessed the PHQ-9 with 6000 patients in 8 primary care clinics and 7 obstetric/gynecology clinics. Using the mental health professional interview as the criterion standard, a PHQ-9 score $\geq 10$ had a sensitivity of $88 \%$ and a specificity of $88 \%$ for major depression. PHQ-9 scores of 5 to 9,10 to 14,15 to 19 , and $\geq 20$ represented mild, moderate, moderately severe, and severe symptoms of depression, respectively.
These characteristics as well as its brevity make the PHQ-9 a useful clinical and research screening tool. If the PHQ-9 indicates possible depression, the diagnosis needs to be clinically confirmed. This questionnaire and the research protocol were approved by the Scottsdale Healthcare Institutional Review Board.

\section{Patients}

The patients were selected using a convenience sample of all adult (aged 18 years or older), English-speaking patients who presented to the Heuser Family Practice Center (FPC) with a chief complaint of headache and agreed to participate. For each patient who entered the study in the headache group, an age- ( \pm 3 years) and sexmatched English-speaking patient was selected as a control from subsequent patients who presented on the same day to the FPC (but not necessarily seen by the same physician) with chief complaints of problems other than headache and agreed to participate in the study. Complaints of control patients included both acute and chronic problems such as high blood pressure, cough, sore throat, diabetes, back pain, dysuria, asthma, hay fever, arthritis, diarrhea, and abdominal pain. Patients were excluded from the control group if any headache diagnosis was listed on the problem list in their medical record. Only English-speaking patients were enrolled in the study; self-reporting tests do not translate well because of semantic differences between languages. ${ }^{12}$ Enrollment continued until the sample number of 200 (100 in the headache group and 100 in the control, or nonheadache, group) was achieved. The enrollment number of 200 was based on the power calculation as discussed in the data analysis section, below. Enrollment occurred from June 2003 through February 2005.

\section{Design}

This was a case-control study. The nursing staff at the FPC identified patients who presented with a chief complaint of headache as well as possible control patients with chief complaints not including headache. The investigators obtained informed consent from interested patients and requested they complete the PHQ-9. The investigators scored the completed PHQ-9. The investigators notified the primary care physician of any study patient who scored $\geq 5$. 
Table 1. Age and Sex of Patients in the Headache and Control Groups $(n=200)$

\begin{tabular}{lccc}
\hline & Total Patients & Headache Group $(\mathrm{n}=100)$ & Control Group* $(\mathrm{n}=100)$ \\
\hline Age, years (mean \pm SD) & $43.8 \pm 15.3$ & $43.7 \pm 15.6$ & $43.9 \pm 15.2$ \\
Sex (n [\%]) & & & $16(16.0)$ \\
Male & $32(16.0)$ & $16(16.0)$ & $84(84.0)$ \\
Female & $168(84.0)$ & $84(84.0)$ & \\
\hline
\end{tabular}

${ }^{*}$ Control group included patients presenting without headache.

\section{Data Analysis}

The age and sex characteristics of the patients as well as the prevalence of reported symptoms of depression (PHQ-9 score $\geq 5$ ) in the patients with headache are reported with descriptive statistics. The proportion of patients who reported symptoms of depression in the headache group was compared with the proportion of patients with reported symptoms of depression in the control (nonheadache) group using the $\chi^{2}$ test. SPSS software version 15.0 (SPSS, Inc., Chicago, IL) was used for all analyses. From previous studies, the prevalence of major depression in the control group was estimated at $10 \%$. Assuming $P<.05$ and $\mathrm{n}=200$, the power to detect an effect size of $15 \%$ ( $\geq 25 \%$ prevalence of symptoms of depression in the headache group as compared with the control group) is $80 \%$.

\section{Results}

A total of 200 patients entered this study: 100 in the headache group and 100 in the control (nonheadache) group. The mean age of participants in the study was 43.8 years, with a range of 18 to 87 years (see Table 1). Women constituted $84 \%$ and men constituted $16 \%$ of the participants.

Those patients who presented with headache had a $63 \%$ prevalence of reporting at least mild symptoms of depression (PHQ-9 score 25 ) compared with a $29 \%$ prevalence in the nonheadache patients (see Table 2). The prevalence of reporting at least moderate symptoms of depression (PHQ-9 score $\geq 10$ ) was $32 \%$ in the headache patients compared with $12 \%$ in the nonheadache patients.

\section{Discussion}

This case-control study revealed a high prevalence on screening of reported symptoms of depression in adult patients who presented to a primary care office with a chief complaint of headache (almost two-thirds of patients) compared with patients presenting with nonheadache chief complaints (less than one-third). Other studies have found the prevalence of depression (with varying definitions of depression) in headache patients to range from as low as $3.4 \%$ to as high as $78 \% .^{3,4,6,7,13-17}$ These were studies of patients in both the inpatient as well as outpatient settings and most were not from primary care practices. Our study agrees with the "depression" prevalence results of Chung and Kraybill ${ }^{3}$ in a similar outpatient primary care setting if one uses reporting at least mild symptoms of depression (PHQ-9 score $\geq 5$ ). Those authors did not study the prevalence of depression in patients in the same setting as nonheadache complaints. The prevalence of reported symptoms of moderate depression (PHQ-9 score $\geq 10$ ) among the nonheadache patients in our study (12\%) was comparable to the quoted prevalence of major depression among primary care patients (10\%).

There is no definitive answer as to why headache and depression may be related. Many physicians are aware of the commonness of depression in patients

Table 2. Prevalence of Symptoms of Depression

\begin{tabular}{|c|c|c|c|c|}
\hline Reported Symptoms of Depression & Headache Group (n [\%]) & Control Group (n [\%]) & $x^{2}$ & $P$ \\
\hline Mild (PHQ $9 \geq 5$ ) & $63(63.0)$ & $29(29.0)$ & 23.27 & $<.0001$ \\
\hline Moderate (PHQ $9 \geq 10$ ) & $32(32.0)$ & $12(12.0)$ & 11.66 & .001 \\
\hline Moderately severe (PHQ $9 \geq 15$ ) & $19(19.0)$ & $4(4.0)$ & 11.05 & .001 \\
\hline Severe (PHQ $9 \geq 20$ ) & $4(4.0)$ & $2(2.0)$ & 0.69 & .407 \\
\hline
\end{tabular}

PHQ, Patient Health Questionnaire. 
with chronic pain. Neurotransmitters are related to the etiology of both disorders. Neurotransmitters that are known to be altered in depression, like serotonin and norepinephrine, are also involved in the gate control mechanism of pain. ${ }^{13,18,19}$ It has been observed that when a patient is treated with an antidepressant, which is designed to alter neurotransmitter levels, both headache and depression improve. ${ }^{20}$ It is also possible that headache and depression are not causally related, but merely coexist because both problems are common. ${ }^{21}$

It would seem prudent for physicians to be aware of the high prevalence of reported symptoms of depression among patients with headache and to consider treating not just the headache, but both problems if depression is confirmed to exist as well. It makes sense that depression may contribute to the amount of suffering associated with headaches. ${ }^{21}$ It is well established that both headache and depression can impair function. If one of the reasons for treatment is to decrease functional impairments, then depression as well as headache pain needs to be addressed. ${ }^{7}$

Physicians also need to remember the high prevalence of depression in patients with headache when considering medications used to treat recurrent headaches. Several of the medications that might be considered, such as $\beta$-blockers, antianxiety agents, and narcotics, may possibly worsen depression. $^{3}$

This study had several limitations. The medical records of patients who had a positive PHQ-9 screening result were not reviewed to determine whether they had an eventual clinical diagnosis of a depressive disorder (eg, major depressive disorder, dysthymic disorder, or depressive disorder not otherwise specified). It would be expected that more patients would have a positive PHQ-9 screening result than would have an eventual diagnosis of a depressive disorder. Patients in the headache group were not analyzed in subsets based on the final classification of their headache (eg, migraine, tension type, cluster, medication overuse, or secondary to another medical condition). Prevalence of reported symptoms of depression might well vary by headache type. Patients in both the headache group and the control group were not evaluated for other pain disorders. Clearly, the presence of another pain disorder could affect the prevalence of reported symptoms of depression.
Many questions warranting further research remain. Do patients with different types of headaches vary in their prevalence of reported symptoms of depression? Do patients with headache and depression view themselves as more disabled than patients with headache but without depression? In patients with migraine headaches and depression, would antidepressants be more effective as prophylaxis than other medications like $\beta$-blockers or antiseizure medications?

\section{Conclusion}

There is a high prevalence of reported symptoms of depression in patients who present to a primary care physician with a chief complaint of headache. All such patients should be screened for depression.

\section{References}

1. US Preventive Services Task Force. Screening for depression: recommendations and rationale. Am Fam Physician 2002;66:647-50.

2. Katon W, Schulberg H. Epidemiology of depression in primary care. Gen Hosp Psychiatry 1992;14:23747.

3. Chung MK, Kraybill DE. Headache: a marker of depression. J Fam Pract 1990;31:360-4.

4. Mitsikostas DD, Thomas AM. Comorbidity of headache and depressive disorders. Cephalgia 1999;19: 211-7.

5. Sheftell FD, Atlas SJ. Migraine and psychiatric comorbidity: from theory and hypotheses to clinical application. Headache 2002;42:934-44.

6. Lipton RB, Hamelsky SW, Kolodner KB, Steiner TJ, Stewart WF. Migraine, quality of life, and depression: a population-based case-control study. Neurology 2000;5 5:629-35.

7. Holroyd KA, Stensland M, Lipchick GL, Hill KR, O'Donnell FS, Cordingley G. Psychosocial correlates and impact of chronic tension-type headaches. Headache 2000;40:3-16.

8. Diamond S. Depression and headache. Headache 1983;23:122-6.

9. Nease DE, Malouin JM. Depression screening: a practical strategy. J Fam Pract 2003;52:118-24.

10. Kroenke K, Spitzer RL, Williams JBW. The PHQ-9: validity of a brief depression severity measure. J Gen Intern Med 2001;16:606-13.

11. Williams JW, Noel PH, Cordes JA, Ramirez G, Pignone $M$. Is this patient clinically depressed? JAMA 2002;287:1160-70.

12. Kerr LK, Kerr LD. Screening tools for depression in primary care. West J Med 2001;175:349-52.

13. Okasha A, Ismail MK, Khalil AH, Fiki RE, Soliman A, Okasha T. A psychiatric study of nonorganic 
chronic headache patients. Psychosomatics 1999;40: 233-8.

14. Yucel B, Kora K, Ozyalcin S, Alcalar N, Ozdemir O, Yucel A. Depression, automatic thoughts, alexithymia, and assertiveness in patients with tension-type headache. Headache 2002;42:194-9.

15. Puca F, Genco S, Prudenzano MP, Savarese M, Bussone G, D'Amico D. Psychiatric comorbidity and psychosocial stress in patients with tension-type headache from headache centers in Italy. The Italian Collaborative Group for the Study of Psychopathological Factors in Primary Headaches. Cephalalgia 1999;19:159-64.

16. Couch JR, Ziegler DK, Hassanein RS. Evaluation of the relationship between migraine headache and depression. Headache 1975;15:41-50.

17. Kashiwagi T, McClure JN, Wetzel RD. Headache and psychiatric disorders. Dis Nerv Syst 1972;33: 659-63.

18. Garvey MJ, Tollefson GD, Schaffer CB. Migraine headaches and depression. Am J Psychiatry 1984; 141:986-8.

19. Garvey MJ, Schaffer CB, Tuason VB. Relationship of headaches to depression. Br J Psychiatry 1983; 143:544-7.

20. Cox D, Thomas D. Relationship between headaches and depression. Headache 1981;21:261-3.

21. Barolin GS. Brief report: headache and depression. Headache 1976;16:252-3. 